\title{
Glaucoma and Diabetes: A Sweet Connection
}

\author{
Chevy Singh ${ }^{1}$, Denis Jusufbegovic ${ }^{2}$, John Lind ${ }^{3}$, Padmanabhan Pattabiraman ${ }^{4}$ \\ ${ }^{1}$ Indiana University School of Medicine; ${ }^{2-4}$ Indiana University School of Medicine, Department of \\ Ophthalmology
}

\section{Background/Objective:}

Glaucoma is the leading cause of irreversible blindness in the world. In the United States alone, a little more than three million people live with this disorder which is caused by damage to the optic nerve found in the back of the eye. This is most commonly caused by an increase in the intraocular pressure, or the fluid that maintains the shape and provides nutrients to the eye. Diabetes mellitus (DM), an age-related disorder, is a risk factor for glaucoma. Post-meal, the sugar one eats is managed in the body by the hormone insulin. However, the excess sugar found in diabetics, can exhaust the production of insulin and lead to many adverse complications in the body as well as increase risk of disease. Although some studies suggest a correlation between diabetes and glaucoma, the mechanisms of this association has not been thoroughly investigated. Our objective is to further explore this connection and provide the mechanisms that link diabetes to glaucoma.

\section{Results:}

From retrospective literature review, we find that diabetes is known to have an additive effect on neural apoptosis and in the generation of reactive oxygen in patients with high intraocular pressure. DM also impairs retrograde axonal transport, causes an accumulation of fibronectin in trabecular meshwork, changes eye vasculature, causes dysregulation in lipid metabolism, and malabsorption of vitamins.

\section{Conclusion \& Scientific/Clinical Policy Impact and Implications:}

This study sheds light on one of the most troubling eye conditions worldwide. The symptoms of diabetes described here increase ones' risk of developing glaucoma. These pathways result in obstruction of aqueous humor drainage or direct damage to the optic nerve cells leading to glaucoma. Diabetes is a common disease that involves many body systems. With knowledge of the relationship between diabetes and glaucoma, measures can be taken to prevent irreversible eye damage in the diabetic population. 